\title{
Coherent response of the Hodgkin-Huxley neuron in the high-input regime
}

\author{
Alessandro Torcini, ${ }^{1,2, * \text { : Stefano Luccioli, }{ }^{1,2} \text { and Thomas Kreuz }}{ }^{1}$ \\ ${ }^{1}$ Istituto dei Sistemi Complessi - CNR, via Madonna del Piano 10, I-50019 Sesto Fiorentino, Italy \\ ${ }^{2}$ INFN Sezione di Firenze, via Sansone, 1 - I-50019 Sesto Fiorentino, Italy
}

\begin{abstract}
We analyze the response of the Hodgkin-Huxley neuron to a large number of uncorrelated stochastic inhibitory and excitatory post-synaptic spike trains. In order to clarify the various mechanisms responsible for noise-induced spike triggering we examine the model in its silent regime. We report the coexistence of two distinct coherence resonances: the first one at low noise is due to the stimulation of correlated subthreshold oscillations; the second one at intermediate noise variances is instead related to the regularization of the emitted spike trains.
\end{abstract}

Keywords: Hodgkin-Huxley model, Coherence, Noise, Sub- and Suprathreshold oscillations

\section{A. Introduction}

In the last decades a large number of studies have been devoted to the characterization of the response of simple and more elaborated neuronal models under the influence of a large variety of stochastic inputs [4, 6, 26]. One of the main reasons justifying the interest of neuroscientists for this subject resides in the observation that in vivo neocortical neurons are subjected to a constant bombardment of excitatory and inhibitory post-synaptic potentials (EPSPs and IPSPs), somehow resembling a background noise [3].

Among the many proposed biophysical models the Hodgkin-Huxley model [5] can still be considered as a reasonably valid framework for exploring the main features of neural dynamics [16]. In order to understand the origin of the variability observed in the distribution of spikes emitted by cortical neurons [23] the response of the Hodgkin-Huxley (HH) model has recently been studied under the influence of additive noise [1, 20, 25] and subjected to trains of post-synaptic potentials $2,0,20,25]$.

One of the most interesting phenomena observed experimentally and numerically for excitable neuronal systems driven by noise is coherence resonance (CR) [8, 17]: it refers to the regularization of noise-induced oscillations occuring for finite (not zero) noise intensity in the absence of any external injected signal (for a comprehensive review see [11]). Evidences of CR have been reported experimentally for the cat's spinal and cortical neural ensembles [15] and theoretically for various neuronal models 11. In particular, $\mathrm{CR}$ has been observed for the $\mathrm{HH}$ model [10, 27], but these results mainly refer to additive continuous noise.

Our aim is to perform a detailed analysis of the response of the $\mathrm{HH}$ model in the silent regime when it is subjected to many (hundreds or thousands) stochastic trains of post-synaptic potentials (PSPs) per emitted spike (i.e. the system is in the so-called high-input regime [3, 23] ).

The different mechanisms responsible for noise-induced

*Electronic address: alessandro.torcini@isc.cnr.it neuronal firing are analyzed in terms of statistical indicators (interspike-interval distributions and their first moments) as well as of dynamical indicators (autocorrelation functions). The analysis of the correlation time reveals a CR associated to the stimulations of coherent subthreshold oscillations around the rest state. This new type of CR coexists with standard CR related to the regularization of spike sequences [1]].

\section{B. Model and methods}

The HH model describes the dynamical evolution of the membrane potential $V(t)$. It can be written as

$$
\begin{aligned}
C \frac{d V}{d t} & =g_{N a} m^{3} h\left(E_{N a}-V\right)+g_{K} n^{4}\left(E_{K}-V\right)+(1) \\
& +g_{L}\left(E_{L}-V\right)+I(t),
\end{aligned}
$$

where $I(t)$ represents the synaptic current and the evolution of the gating variables $X=m, n, h$ is ruled by three ordinary differential equations of the form $d X / d t=$ $\alpha_{X}(V)(1-X)-\beta_{X}(V) X$. The parameters entering in Eq. (11) and the expressions of the nonlinear functions $\alpha_{X}(V)$ and $\beta_{X}(V)$ are reported in [12].

In this study we consider the response of the single $\mathrm{HH}$ model to $N_{e}$ (resp. $N_{i}$ ) uncorrelated trains of EPSPs (resp. IPSPs) with $N_{e}\left(N_{i}\right)$ ranging from 10 to 10,000. By following Refs. 2, 7], each PSP is schematized as an instantaneous variation of the membrane potential by a positive (resp. negative) amount $\Delta V$ for excitatory (resp. inhibitory) synapses. The amplitude of each voltage kick is assumed to be $0.5 \mathrm{mV}$, i.e. reasonably small $(\approx 7 \%)$ with respect to the distance between the "threshold" for spike initiation for rapid EPSPs and the resting potential $(\approx 6-7 \mathrm{mV})$ estimated for the HH model $13,[26]$. Moreover, amplitudes $\approx 0.5 \mathrm{mV}$ are comparable with average EPSPs experimentally measured for pyramidal neurons in the visual cortex of rats $[\underline{6}]$. This amounts to exciting the neuron (1) with an impulsive current

$$
I(t)=Q\left[\sum_{k=1}^{N_{e}} \sum_{l} \delta\left(t-t_{k}^{l}\right)-\sum_{m=1}^{N_{i}} \sum_{n} \delta\left(t-t_{m}^{n}\right)\right]
$$


where $t_{k}^{l}$ (resp. $t_{m}^{n}$ ) are the arrival times of the EPSPs (resp. IPSPs) and $Q=C \Delta V$ is the charge associated to each kick. In order to mimic the inputs received by cortical neurons, for each afferent (excitatory and inhibitory) synapse the time interval distribution between PSP inputs is chosen to be Poissonian with an average frequency $\nu_{0}=100 \mathrm{~Hz}[23]^{1}$.

The stochastic input can be characterized in terms of the average and the variance of the net spike count within a temporal window $\Delta T$

$$
N(\Delta T)=\sum_{k=1}^{N_{e}} n_{k}^{E}(\Delta T)-\sum_{m=1}^{N_{i}} n_{m}^{I}(\Delta T)
$$

where $n_{k}^{E}(\Delta T)$ (resp. $\left.n_{m}^{I}(\Delta T)\right)$ represents the number of afferent EPSPs (resp. IPSPs) received from neuron $k$ (resp. $m$ ) in the interval $\Delta T$. In the high input regime, by assuming uncorrelated input spike trains, $N(\Delta T)$ follows a Gaussian distribution (cf. [12]), with average and variance derivable within the shot noise formalism as

$$
<N(\Delta T)>=\left(N_{e}-N_{i}\right) \Delta T \nu_{0}
$$

and

$$
\operatorname{Var}[N(\Delta T)]=\left(N_{e}+N_{i}\right) \Delta T \nu_{0}=\sigma^{2} \Delta T \nu_{0}
$$

The parameter $\sigma=\sqrt{\left(N_{e}+N_{i}\right)}$ measures the standard deviation of the stochastic input process.

The average current stimulating the neuron is given by

$$
\bar{I}=\frac{C \Delta V<N(\Delta T)>}{\Delta T}=C \Delta V \nu_{0}\left(N_{e}-N_{i}\right) .
$$

$\bar{I}$ represents the bifurcation parameter ruling the dynamics of the deterministic $\mathrm{HH}$ model; for $\bar{I}<I_{S N} \simeq$ $6.27 \mu \mathrm{A} / \mathrm{cm}^{2}$ the model is in the silent regime, i.e., in the absence of noise the dynamics is attracted towards a stable fixed point and the neuron does not fire. However, since the fixed point is a focus the relaxation towards the rest state occurs via damped oscillations (subthreshold oscillations) [19]. Periodic firing is observed for the $\mathrm{HH}$ model only above $I_{S N}$ and it is associated to frequencies in the range $50-80 \mathrm{~Hz}$.

We have verified that in the high input regime the response of the neuron (for fixed $\nu_{0}$ ) is determined once fixed $\bar{I}$ and $\sigma$, therefore it does not depend separately on $N_{e}$ and $N_{i}$, but only on their difference $\left(N_{e}-N_{i}\right) \propto \bar{I}$ and $\operatorname{sum}\left(N_{e}+N_{i}\right) \propto \sigma^{2}[12]$.

In order to characterize the output of the neuron and to examine the coherence effects in the response we have employed the following indicators: the distribution of the output interspike intervals $P_{I S I}(t)$ and its first moments;

\footnotetext{
1 It should be stressed that the model here analyzed is driven by impulsive post-synaptic currents and it should not be confused with conductance-driven models [20, 25].
}

the coefficient of variation of the output interpike intervals distribution $\left(P_{I S I}(t)\right)$ defined as $R=S_{I S I} / A_{I S I}$, where $A_{I S I}$ is the average and $S_{I S I}$ the standard deviation of the $P_{I S I}(t)$ (for a perfectly periodic response we have $R=0$ and $R=1$ for Poissonian output; the normalized autocorrelation function $C(t)$ for the membrane potential and the correlation time [17] defined as

$$
\tau_{c}=\int_{0}^{\infty} C^{2}(t) d t
$$

CR is usually identified by a minimum (resp. a maximum) in the coefficient of variation $R$ (resp. in $\tau_{c}$ ) occurring at finite noise variance [1].

\section{Results}

The HH neuron subjected to stochastic input exhibits a noise-induced (irregular) spiking behaviour with an average firing rate $\nu_{\text {out }}=1 / A_{I S I}$. For fixed $\bar{I}$ the firing rate increases with the standard deviation of the noise (cf. Fig. 1).

The noise-induced firing activity becomes more and more pronounced by approaching the repetetive firing bifurcation $I_{S N}$ for the deterministic $\mathrm{HH}$. The $\mathrm{HH}$ model is a type II neuron, therefore the onset of oscillation at $I \simeq I_{S N}$ is associated with a finite frequency $(\simeq 50 \mathrm{~Hz})$, however, in presence of noise, arbitrarily low spike rates can be observed even in the silent regime.

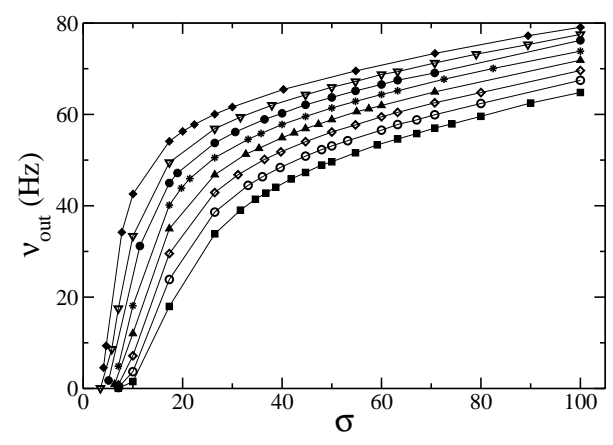

FIG. 1: Silent regime: average firing rate $\nu_{\text {out }}$ as a function of $\sigma$ for increasing values of $\bar{I}$ (from the bottom to the top $\left.\bar{I}=-1,0,1,2,3,4,5,6.15 \mu \mathrm{A} / \mathrm{cm}^{2}\right)$.

\section{Response of the HH model in the low and high noise limit}

Let us now examine more in detail the origin of noiseinduced spiking in the low and high noise limits. For low noise we observe the coexistence of multi-peaks and an exponential tail in the $P_{I S I}(t)$ (cf. Fig. 2). As explained in the following the multi-peak structure is related to the relaxation dynamics of the membrane potential $V(t)$ 
following a spike emission, while the exponential tail is associated with noise induced activation processes.

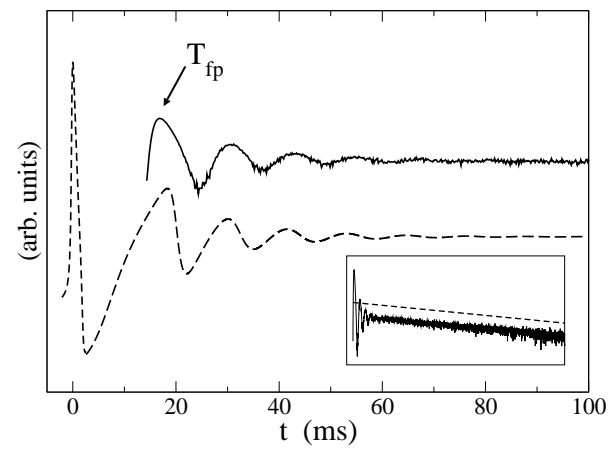

FIG. 2: $P_{I S I}(t)$ obtained for the stochastic input (2) with $\sigma=4.6$ (continuous curve) and the relaxation dynamics of the membrane potential $V(t)$ following a step current stimulation (dashed line). The position of the output spike has been shifted to $t=0$ and the action potential has been rescaled to better reveal the relaxation oscillations. In the inset the exponential tail due to the activation mechanism is shown. The data refer to $\bar{I}=6.15 \mu \mathrm{A} / \mathrm{cm}^{2}$.

To better understand the first mechanism we have studied the evolution of $V(t)$ following a step current of amplitude $\bar{I}$, i.e.,

$$
I(t)=\left\{\begin{array}{ll}
0 & \text { if } t \leq 0 \\
\bar{I} & \text { if } t>0
\end{array} .\right.
$$

As shown in Fig 2 (dashed line) $V(t)$ exhibits one spike followed by damped oscillations. This suggests that the probability of eliciting a second spike is enhanced in correspondence the maxima of the relaxation oscillations, where $V(t)$ is nearest to the firing threshold. Moreover, it is possible to show that the period $T_{n l}$ of the first oscillation has a clear nonlinear origin, while the period of successives oscillations $T_{l}$ can be explained by a linear stability analysis around the fixed point. Indeed we have verified that the first peak $\left(T_{f p}\right)$ observed in the distribution $P_{I S I}(t)$ (continuous curve in Fig 2) is related to $T_{n l}$, while the successives peaks in the $P_{I S I}(t)$ correspond to the linear oscillations of period $T_{l}$ [12].

The second firing mechanism responsible for the exponential tail in the $P_{I S I}(t)$ is due to the competition between two effects, on one side the tendency of the dynamics to relax towards its stable fixed point and on the other side noise fluctuations that instead lead the system towards the firing threshold. In this framework the dynamics of $V(t)$ can be described as the overdamped dynamics of a particle in a potential well under the influence of thermal fluctuations [14] and the average firing time, or activation time $T_{a}$, can be expressed in terms of the Kramers expression 14 for sufficiently small noise:

$$
T_{a} \propto \mathrm{e}^{W / \sigma^{2}},
$$

where $\sigma^{2}$ plays the role of an effective temperature and $W$ of an energy barrier. For $\sigma^{2}<W$, i.e., in the low noise limit, the dynamics can be characterized as an activation process: $P_{I S I}(t)$ resembles a Poissonian distribution with $R \approx 1$. For $\sigma^{2}>W$, i.e., in the high noise limit, the multi-peak structure and the exponential tail disappear and $P_{I S I}(t)$ reduces to an inverse Gaussian corresponding to the distribution of the first passage times for a diffusive process plus drift [26].

As a further indicator we have estimated the spike triggered average potential (STAP) [18], that gives the average shape of $V(t)$ preceding the emission of a spike, for sufficiently long ISIs and for small $\sigma$. As shown in Fig. [3] $V(t)$ oscillates with period $\approx T_{l}$ before firing; therefore the emission of a spike (for long ISIs) is triggered by the excitation of linear subthreshold oscillations around the fixed point. Thus the HH neuron nearby the rest state acts as a sort of selective filter since it responds (by emitting a spike) with higher probability when it is stimulated with a specific input frequency $\left(\approx 1 / T_{l}=61-88 \mathrm{~Hz}\right.$ for $\left.0 \leq \bar{I} \leq I_{S N}\right)$. This result agrees with the analysis of [27] where it has been shown that a silent HH neuron subjected to a sinusoidal current optimally resonates when forced with a frequency linearly correlated with $1 / T_{l}$.

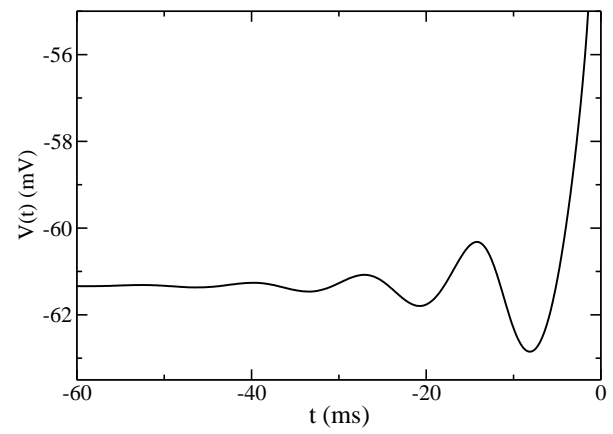

FIG. 3: $\bar{I}=5 \mu \mathrm{A} / \mathrm{cm}^{2}$ and $\sigma=5.7$. STAP preceding the emission of a spike. At time $t=0$ the potential overcomes the spike detection threshold (fixed at $-5 \mathrm{mV}$ ). STAP has been calculated by averaging only over ISIs longer than 150 ms.

\section{Coherent response of the Hodgkin-Huxley neuron}

In the silent regime we have found the coexistence of two CRs: one is related to the regularization of the emitted spike trains at intermediate noise levels and has previously been reported in [10, 27]; the second one, observed for the first time, occurs at very low noise and is associated to the coherence of subthreshold oscillations.

The first CR can be explained by noticing that the dynamics of excitable systems can be characterized in terms of two characteristic times [17]: an activation time $T_{a}$, representing the time needed to excite the system, and an excursion time $T_{e}$, indicating the duration of the spike (i.e., the time spent in the excited state). An ISI is given by the sum of these two times and therefore $R$ can 
be (formally) expressed as the sum of two parts depending separately on $T_{a}$ and $T_{e}$. These two contributions vary in an opposite way when the noise is increased and the competition of these two tendencies leads to the maximal coherence (associated to a minimum in $R$ ) for finite noise [11, 17]. So there is an initial range of $\sigma$-values where by increasing the noise the spike emission is facilitated ( $T_{a}$ reduces accordingly to Eq. (9)) and this renders the response of the neuron more and more regular. On the other side at high $\sigma$-values the response becomes again more irregular, because the noise induces firing even during the relative refractory period thus modifying even the duration $T_{e}$ of the spike itself. To summarize, the activation process responsible for firing is gradually substituted by a diffusive process with drift and at the transition from one mechanism to the other a regularization of the output signal is observed. As shown in Fig. 4. for different values of $\bar{I}$, both the coefficient of variation $R$ and the signal correlation time $\tau_{c}$ were able to identify clearly the first kind of coherence in the silent regime. We can also see that $\tau_{c}$ increases and $R$ decreases when $\bar{I} \rightarrow I_{S N}$, i.e., the coherence of the emitted spike trains increases by approaching the repetetive firing regime.

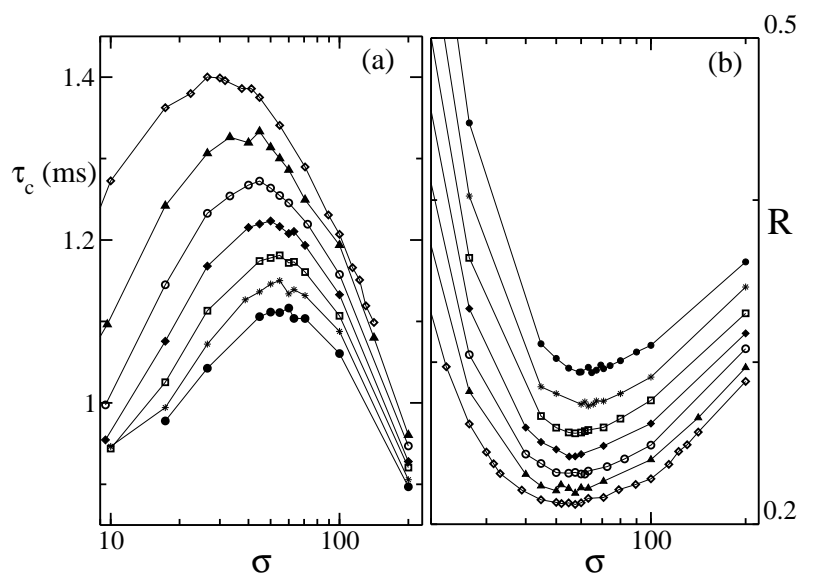

FIG. 4: Coherence of the emitted spike trains for increasing values of $\bar{I}$ in the silent regime. (a) $\tau_{c}$ (from bottom to top $\bar{I}=-1,0,1,2,3,4,5 \mu \mathrm{A} / \mathrm{cm}^{2}$ ). (b) $R$ (from top to bottom $\bar{I}=-$ $\left.1,0,1,2,3,4,5 \mu \mathrm{A} / \mathrm{cm}^{2}\right)$.

The second kind of resonance could only be detected by $\tau_{c}$. In fact this property is not related to spike emission (suprathreshold dynamics) but to the behaviour of the signal below the firing threshold. In Fig 5 the behaviour of $\tau_{c}$ for $\bar{I}=4 \mu \mathrm{A} / \mathrm{cm}^{2}$ is shown in a wider range of noise with respect to Fig. 4h. In this range $\tau_{c}$ exhibits indeed two maxima: the first and higher maximum at low noise $(\sigma \approx 3)$ is related to the coherence of subthreshold oscillations, while the second one at intermediate noise $(\sigma \approx 33)$ is due to the CR just discussed above.

Let us now gain deeper insight about the origin of these two maxima. For extremely low noise $(\sigma<3)$ the neuron rarely fires, i.e., $T_{a} \rightarrow \infty$; increasing the noise the subthreshold oscillations generated by the input kicks are
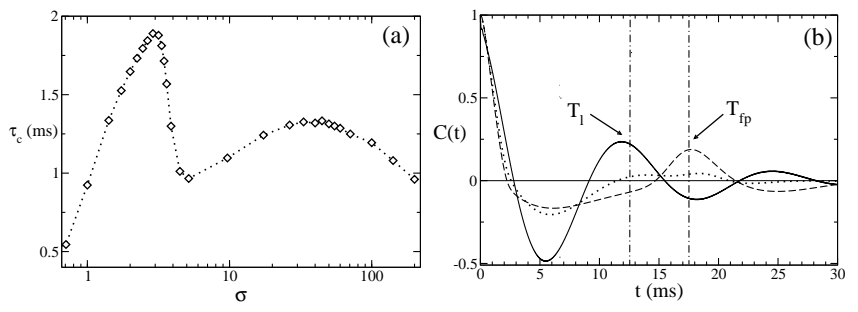

FIG. 5: $\bar{I}=4 \mu \mathrm{A} / \mathrm{cm}^{2}$ : (a) correlation time $\tau_{c}$ (empty circles) as a function of the standard deviation of the input noise $\sigma$ : (b) autocorrelation function $C(t)$ for three increasing noise values $\sigma=2.9$ (continuous line), 4.5 (dotted line) and 9.7 (dashed line); the vertical dot-dashed lines indicates the pe$\operatorname{riod} T_{l}$ and the period $T_{f p}$ for $\sigma=9.7$.

more and more correlated. For $\sigma>3$ the occurrence of rare spikes tends to decorrelate the signal leading to a decrease of $\tau_{c}$. With a further increase of the noise $V(t)$ becomes essentially a sequence of spikes and $C(t)$ represents the correlation between them; a second peak appears in $\tau_{c}$ indicating the maximal coherence of the spike sequence.

To investigate the transition from one to the other behaviour let us analyze the autocorrelation function of $V(t)$ for increasing value of $\sigma$, as shown in Fig. 5b. For $\sigma \approx 3$ the autocorrelation function $C(t)$ oscillates with a period $\approx T_{l}$ (see Sec C1), while at $\sigma \approx 9.7$ the maxima of $C(t)$ correspond to multiples of $T_{f p}$, i.e., the first peak of $P_{I S I}(t)$. In between these two values there is a transition where the course of $V(t)$, initially consisting of mere subthreshold oscillations, is dominated by the spikes. The transition is observed for $\sigma \approx 4.5$, where in correspondence to the first oscillation $C(t)$ reveals two maxima, one located at $t \approx T_{l}$ and another at $t \approx T_{f p}$.

\section{Conclusions}

We have presented an analysis of the response of the silent $\mathrm{HH}$ model in the high-input regime. In this regime the $\mathrm{HH}$ neuron displays a large variety of dynamical behaviors and its response is completely determined by the average and the variance of the stochastic input. Our main result is the coexistence of two different coherence resonances: the first one, at intermediate noise levels, associated with the regularization of the spikes emitted by the neuron; the second one, at very low noise, related to the coherence of subthreshold oscillations around the fixed point. The first one can be revealed using both the ISI coefficent of variation $R$ and the autocorrelation time of the signal $\tau_{c}$. The second type of resonance, observed for the first time, is not related to spike emission and can thus only be detected by means of $\tau_{c}$.

Moreover, we have examined the behaviour of the $\mathrm{HH}$ neuron for low and high input noise variance. The richness of the $\mathrm{HH}$ dynamics is particularly pronounced for 
low input noise where we have found the coexistence of a multimodal structure and an exponential tail in the $P_{I S I}(t)$. The peaks in the $P_{I S I}(t)$ suggests that the system, under the influence of stochastic inputs, can resonate when forced with specific frequencies: the first peak is associated to frequencies in the $\gamma$-range 24] (namely, from 40 to $66 \mathrm{~Hz}$ for $\bar{I} \in[0: 9] \mu A / \mathrm{cm}^{2}$ ), and the second one to lower frequencies (namely, from 30 to $37 \mathrm{~Hz}$ for the same interval of currents) 12 .

In the silent regime the responsiveness of the single neuron is enhanced by stochastic stimulations, in particular the regularity of the emitted spike trains is maximal in correspondence to an optimal noise level. Moreover, we expect that a population of such neuronal elements will have the capability to exhibit coherent and correlated activity over different time scales (mainly in the $\gamma$ and $\beta$-ranges [24]), a property that is believed to be important for information encoding for cortical processing [22]. Indeed it has been found [27] that for sufficiently strong synaptic coupling a globally coupled $\mathrm{HH}$ network subjected to stochastic inputs reveals a collective synchronized rhythmic firing in a range of $40-60$ $\mathrm{Hz}$, induced via $\mathrm{CR}$.

To proceed in the direction of more realistic situations the present analysis performed for a current-driven model should be extended by considering conductance-based synaptic inputs [20, 25]. It is surely worth to address this issue in forthcoming studies, because the response of these two classes of models to noise fluctuations can sometimes be even opposite, as shown in 25. for the output firing rate.
[1] B. Agüera y Arcas, A.L. Fairhall, and W. Bialek, Computation in a single neuron: Hodgkin-Huxley revisited, Neural Computation 15, (2003) 1715-1749.

[2] D. Brown, J. Feng and S. Feerick, Variability of Firing of Hodgkin-Huxley and FitzHugh-Nagumo Neurons with Stochastic Synaptic Input, Phys. Rev. Lett. 82 (1999) 4731-4734.

[3] A. Destexhe, M. Rudolph and D. Paré, The highconductance state of neocortical neurons in vivo, Nature Rev. Neurosci. 4, (2003) 739-751.

[4] W. Gerstner and W. Kistler, Spiking Neuron Models, (Cambridge University Press, Cambridge, 2002).

[5] A.L. Hodgkin and A.F. Huxley, A qualitative description of membrane current and its application to conduction and excitation in nerve, J. Physiol. (London) 117 (1952) 500-544.

[6] C. Koch, Biophysics of computation, (Oxford University Press, New York, 1999).

[7] J. Feng and P. Zhang, Behavior of integrate-and-fire and Hodgkin-Huxley models with correlated inputs, Phys. Rev. E, 63 (2001) 051902.

[8] H. Gang, T. Ditzinger, C.Z. Ning, and H. Haken, Stochastic resonance without external periodic force, Phys. Rev. Lett. 71 (1993) 807-810.

[9] H. Hasegawa, Responses of a Hodgkin-Huxley neuron to various types of spike-train inputs, Phys. Rev. E 61 (2000) 718-726.

[10] S.G Lee, A. Neiman, and S. Kim, Coherence resonance in a Hodgkin-Huxley neuron, Phys. Rev. E 57 (1998) 3292-3297.

[11] B. Lindner, J. Garcìa Ojalvo, A. Neiman, and L. Schimansky-Geier, Effects of noise in excitable systems, Physics Reports 392, (2004) 321-424.

[12] S. Luccioli, T. Kreuz, and A. Torcini, Dynamical response of the Hodgkin-Huxley model in the high-input regime, Phys. Rev. E 73 (2006) 041902.

[13] D. Noble and R.B. Stein, The threshold conditions for initiation of action potentials by excitable cells, J. Physiol. 187 (1966) 129-161.

[14] N.G. van Kampen, Stochastic Processes in Physics and Chemistry, Elsevier, North Holland, (2001)

[15] E. Manjarrez , J.G. Rojas-Piloni, I. Méndez, L. Martínez, D. Vélez, D. Vázquez and A. Flores, Internal stochastic resonance in the coherence between spinal and cortical neuronal ensembles in the cat, Neurosci. Lett. 326 (2002) 93-96.

[16] C. Meunier and I. Segev, Playing the devil's advocate: is the Hodgkin-Huxley model useful?, TRENDS Neurosci. 25 (2002) 558-63.

[17] A.S. Pikovsky and J. Kurths, Coherence Resonance in a Noise-Driven Excitable System, Phys. Rev. Lett. 78 (1997) 775-778.

[18] F. Rieke, D. Warland, Rob de Ruyter van Steveninck e W. Bialek, Spikes: exploring the neural code, MIT: Cambridge, Massachusetts (1996).

[19] J. Rinzel and R. Miller, Numerical calculation of stable and unstable periodic solutions to the Hodgkin-Huxley equations, Math. Biosci. 49 (1980) 27-59.

[20] M. Rudolph and A. Destexhe, The discharge variability of neocortical neurons during high-conductance state, Neuroscience 119 (2003) 855-873

[21] E. Salinas and T.J. Sejnowski, Impact of correlated synaptic input on output firing rate and variability in simple neuronal models, The Journal of Neuroscience 20 (2000) 6193-6209.

[22] E. Salinas and T.J. Sejnowski, Correlated neuronal activity and the flow of neural information, Nature Rev. Neurosci. 2 (2001) 539-550.

[23] M.N. Shadlen and W.T. Newsome, The variable discharge of cortical neurons: implications for connectivity, computation and information coding, J. Neurosci. 18, (1998) 3870-3896.

[24] The synaptic organization of the brain, ed. G.M. Sheperd (Oxford University Press, New York, 2004)

[25] P.H.E. Tiesinga, J.V. Josè, and T.J. Sejnowski, Comparison of current-driven and conductance-driven neocortical model neurons with Hodgkin-Huxley voltage-gated channels, Phys. Rev. E 62, (2000) 8413-8419

[26] H.C Tuckwell, Introduction to theoretical neurobiology, (Cambridge University Press, New York, 1988).

[27] Y. Yu, W. Wang, J. Wang, and F. Liu, Resonanceenhanced signal detection and transduction in the Hodgkin-Huxley neuronal systems, Phys. Rev. E 63, (2001) 021907. 